\title{
Research on Modeling and Experiment of Glass Substrate Peeling Based on Adhesion Theory
}

\author{
Liwei HOU, Hengsheng WANG, Runhua LIU
}

\begin{abstract}
In this paper, the peeling of glass substrates is modeled, in a setting of automatic polishing and grinding for flat panel display glass substrates. The mechanical model of glass substrates-adhesive pad structure is established. The vacuum adsorbing force between them is regarded as adhesive force. The model is simplified as a distributed spring group which can describe the desorption and shear behavior of the glass substrates-adhesive pad structure. The corresponding analytical solution method is proposed. Finally, experiment is conducted to verify the accuracy and feasibility of the proposed mechanical model.
\end{abstract}

Keywords: desorption; glass substrate; mechanical model; vacuum adsorbing force

\section{INTRODUCTION}

Flat Panel Display (FPD) is another large-scale industry developed after integrated circuit (IC). Along with IC, FPD has become the core and foundation of electronic information industry. With the rapid development of flat panel display industry, the use of flat panel display glass substrates is also increasing rapidly. At present, the thickness of glass substrates used in commerce is usually less than $0.7 \mathrm{~mm}$, which requires thinning process to achieve the purpose of thinning. Chemical Mechanical Polishing (CMP) technology is the most widely used thinning process. Considering the particularity of the process, glass substrates are usually fixed on the grinding and polishing machine by polyurethane adsorption pad. At present, all the polishing and abrasive cutting is operated manually and occupies the whole process for a long time. The realization of automatic cutting system is an inevitable choice to improve product quality consistency and production efficiency of glass substrates. In order to realize automatic polishing and cutting, in other words, to achieve the desorption of glass substrates, it is necessary to accurately describe the mechanical behavior between the adsorbent pad and glass substrates, which is the key to achieve non-destructive desorption and improve production efficiency simultaneously.

The adsorption between the adsorbent pad and the glass substrate is similar to many natural phenomena, such as insects adsorbing on the surface of the object, geckos walking on the vertical wall or even on the ceiling. These phenomena are difficult to explain by the classical contact theory, because the influence of material surface effect on the contact performance is not considered. For this reason, many scholars have developed many contact mechanics models based on continuum mechanics, which can consider the surface effect of materials. However, these models all adopt the near-spherical hypothesis without exception. These models have great limitations in describing the adsorption behavior of adsorption pads on glass substrates. Igor Sokolov [5] applied Atomic Force Microscope (AFM) to detect the morphology of nano-area on the surface of polyurethane adsorbent pad, and measured its mechanical properties (elasticity and viscidity). Atomic force microscopy is helpful to analyze the adsorption mechanism of polyurethane adsorbent pad on glass substrates, but it is difficult to reflect the mechanical behavior of adsorption and desorption of polyurethane adsorbent pad and polyurethane adsorbent pad. At present, the research on adsorbing glass substrates by adsorbing pads only appears in practical engineering applications, and the adsorbing force is basically measured by experiments. Therefore, it is important to establish a more general mathematical model for the stripping of glass substrates from the adsorbent pad, which can provide a mechanical theoretical basis for the analysis of the automatic feeding and unloading process of glass substrates.

The failure of bonding interface based on fracture mechanics can describe the process of glass substrate desorption more accurately. In this paper, the process of non-destructive peeling of glass substrate is modeled based on bonding theory, which provides theoretical support for the automatic production of glass substrate thinning process. The structure of this paper is as follows: the first part analyses the research background and key problems to be solved; the second part analyses the process of glass substrate peeling and builds the model based on the bonding theory; the third part solves the mechanical model; and the fourth part verifies the mechanical model.

\section{MECHANICAL MODELING OF GLASS SUBSTRATE DESORPTION PROCESS}

\subsection{Analysis of Glass Substrate Desorption Process}

The automatic cutting process of glass substrate is shown in Fig. 1. The wedge clamp is inserted into the interface between the glass substrate and the adsorption pad, and then lifted upward to complete the initial peeling of the glass substrate from the surface of the adsorption pad

The wedge mechanism contacts with the bottom surface of the glass substrate and produces a concentrated load to desorb the glass substrate. Because the glass substrate is prone to rupture, the mechanical model of the glass substrate-adsorption pad structure must be established, which plays a decisive role in the nondestructive cutting of the glass substrate. This paper focuses on the modeling of the desorption process of the glass substrate-adsorption pad structure, which is stable and affected by many factors, such as the geometrical size of the glass substrate. Because of the large size of the 
research object, the macro-forces such as gravity and atmospheric pressure are the main influencing factors, while the influence of low-scale adhesion forces such as van der Waals force and surface tension is neglected.

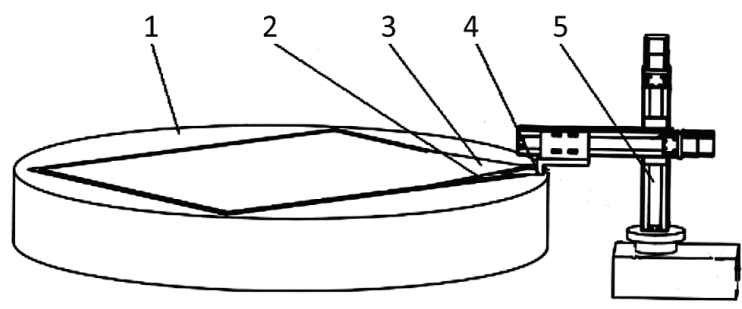

Figure 1 Desorption Process of Glass Substrate: 1 - Bottom fixing plate of polishing machine; 2 - Adsorption pad; 3 - Glass substrate; 4 - Wedge mechanism; 5 - Robotic arm.

\subsection{Modeling Idea}

The glass substrate is tightly attached to the adsorbent pad under vacuum adsorption. This structure can be regarded as a typical sandwich bonding structure, that is, a thin layer of bonding material is sandwiched between the upper and lower layers of the adhesives. The vacuum adsorbing force between the glass substrate and the adsorbing pad is equivalent to the adhesive force of the adsorbing material. Then the desorption process of the glass substrate can be modeled according to the classical Newtonian mechanics. According to this idea, the model is simplified as shown in Fig. 2. The vacuum adsorption between glass substrate and adsorption pad was modeled by assuming a vacuum layer.

The cornerstone of mechanical modeling lies in reasonable assumptions, and the differential equations derived under different assumptions are generally different. The number of constraints of hypothetical conditions should be determined by the objects actually described. The reasonable and satisfying model for engineering needs is the pursuit of theoretical analysis. The hypothesis of vacuum layer modelling includes whether the thickness of vacuum layer is taken into account and whether the shear effect of vacuum layer is taken into account. Based on these assumptions, different vacuum layer models can be obtained, which can be introduced into the constitutive equation of the layer structure, and the differential equation with different complexity can be derived. Considering that the thickness of the vacuum layer can be neglected, the effect of the thickness of the vacuum layer on the transverse distribution of stress is neglected, and the bonding stress along the thickness direction of the vacuum layer is assumed to be constant.

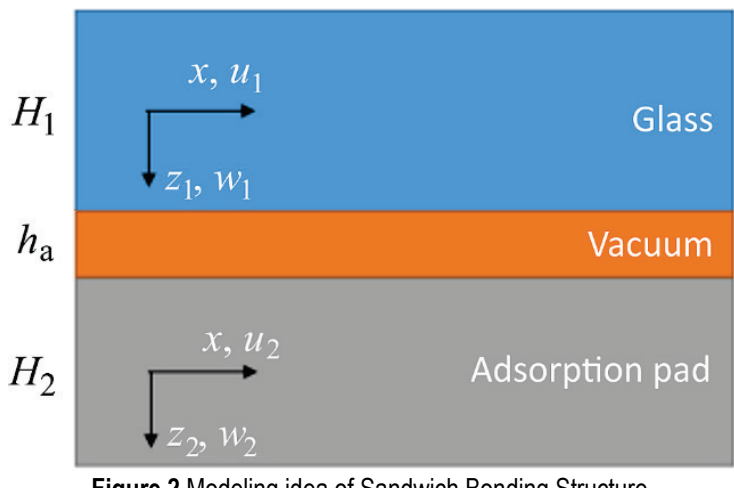

Figure 2 Modeling idea of Sandwich Bonding Structure

In this paper, a spring model is used to simulate the process of crack propagation in the desorption process of glass substrate-adsorbent cushion structure, so as to conveniently study the process of glass peeling and its influence on the fracture parameters such as energy release rate of peeling, which can intuitively reveal the technological principle of glass peeling.

\subsection{Vacuum Layer Model}

According to the deformation theory of Timoshenko beam, the axial and transverse displacement fields in the bonded layer can be expressed as $U_{i}\left(x_{i}, z_{i}\right)=u_{i}(x)+z_{i} \varphi_{i}(x)$ and $W_{i}\left(x_{i}, z_{i}\right)=w_{i}(x)$. Under the assumption that the stress and strain of the vacuum layer change along $Z$ direction, the strain at any point in the vacuum layer can be calculated by the ratio of the relative displacement of the lower edge of glass to the upper edge of the adsorption pad and the thickness of the vacuum layer:

$$
\left\{\begin{array}{l}
\varepsilon_{z z}^{\mathrm{a}}=\frac{W_{2}^{\text {top }}-W_{1}^{\text {bottom }}}{h_{\mathrm{a}}}=\frac{\left.W_{2}\right|_{z_{2}=-h_{2}}-\left.W_{1}\right|_{z_{1}=-h_{1}}}{h_{\mathrm{a}}}=\frac{w_{2}-w_{1}}{h_{\mathrm{a}}} \\
\gamma_{x z}^{\mathrm{a}}=\frac{U_{2}^{\text {top }}-U_{1}^{\text {bottom }}}{h_{\mathrm{a}}}=\frac{\left.U_{2}\right|_{z_{2}=-h_{2}}-\left.U_{1}\right|_{z_{1}=-h_{1}}}{h_{\mathrm{a}}}=\frac{u_{2}-u_{1}-h_{1} \varphi_{1}-h_{2} \varphi_{2}}{h_{\mathrm{a}}}
\end{array}\right.
$$

Among them, $H_{1}$ and $H_{2}$ represent half of the thickness of glass substrate and adsorbent pad respectively. Under the assumption of plane strain, only the transverse normal stress and the longitudinal shear stress of the vacuum layer are considered. According to the linear Hooke's law, the stress combination of vacuum layer is as $\sigma=E_{\mathrm{a}}^{*} \varepsilon_{z z}^{\mathrm{a}}$ and $\tau=G_{\mathrm{a}} \gamma_{x z}^{\mathrm{a}}$, combined with Eq. (1):

$$
\begin{aligned}
& \sigma=E_{\mathrm{a}}^{*} / h_{\mathrm{a}}\left(w_{2}-w_{1}\right) \\
& \tau=G_{\mathrm{a}} / h_{\mathrm{a}}\left(u_{2}-u_{1}-h_{1} \varphi_{1}-h_{2} \varphi_{2}\right)
\end{aligned}
$$

$$
E_{\mathrm{a}}^{*}=E_{\mathrm{a}} /\left(1-v_{\mathrm{a}}^{2}\right), G_{\mathrm{a}}=E_{\mathrm{a}} /\left[2\left(1+v_{\mathrm{a}}\right)\right], \text { representing }
$$
the elastic modulus and shear modulus of a vacuum layer under the assumption of plane strain respectively.

Considering that the vacuum layer is composed of springs uniformly distributed in the transverse direction and the longitudinal direction, the corresponding elastic coefficient is $k_{\sigma}$ and $k_{\tau}$. When the structure is deformed, the amount of deformation of the spring is the horizontal and longitudinal stretching or compression of the upper and lower layers. Therefore, according to Hooke's law, the stress on the spring is: 


$$
\begin{aligned}
& \sigma=k_{\sigma} \Delta W \\
& \tau=k_{\tau} \Delta U
\end{aligned}
$$

Obviously, the relative deformation of the lateral and axial displacement at the interface can be expressed as $\Delta W=W_{2}^{\text {top }}-W_{1}^{\text {bottom }}$ and $\Delta U=U_{2}^{\text {top }}-U_{1}^{\text {bottom }}$. So we have $k_{\sigma}=E_{\mathrm{a}}^{*} / \mathrm{h}_{\mathrm{a}}$ and $k_{\tau}=G_{\mathrm{a}} / \mathrm{h}_{\mathrm{a}}$.

We define the desorption and shear stiffness of the bonding layer, so the desorption and shear stress of the vacuum layer can be expressed as:

$$
\begin{aligned}
& \sigma=k_{\sigma}\left(w_{2}-w_{1}\right) \\
& \tau=k_{r}\left(u_{2}-u_{1}-h_{1} \varphi_{1}-h_{2} \varphi_{2}\right)
\end{aligned}
$$

The desorption stress of the glass substrateadsorption pad depends on the desorption stiffness and the lateral displacement difference between the glass substrate and the neutral surface of the adsorption pad, regardless of the thickness of the upper and lower layers. The shear stress is related not only to the shear stiffness and longitudinal displacement difference, but also to the product of the angle of the interlayer and the half thickness.

\subsection{Equilibrium Equation}

The Fig. 3 is a schematic diagram of the force analysis of the micro-body of the glass substrate-vacuum layeradsorption pad structure. $N_{i}, Q_{i}$, and $M_{i}$ in the Fig. 3 represent the axial force, the transverse shear force and the bending moment load of the unit width structure, respectively. According to the continuous rule of Timoshenko beam, these internal forces can be expressed by displacement and rotation angle:

$$
N_{i}=A_{i} \frac{\mathrm{d} u_{i}}{\mathrm{~d} x}, Q_{i}=C_{i}\left(\frac{\mathrm{d} w_{i}}{\mathrm{~d} x}+\varphi_{i}\right), M_{i}=D_{i} \frac{\mathrm{d} \varphi_{i}}{\mathrm{~d} x}
$$

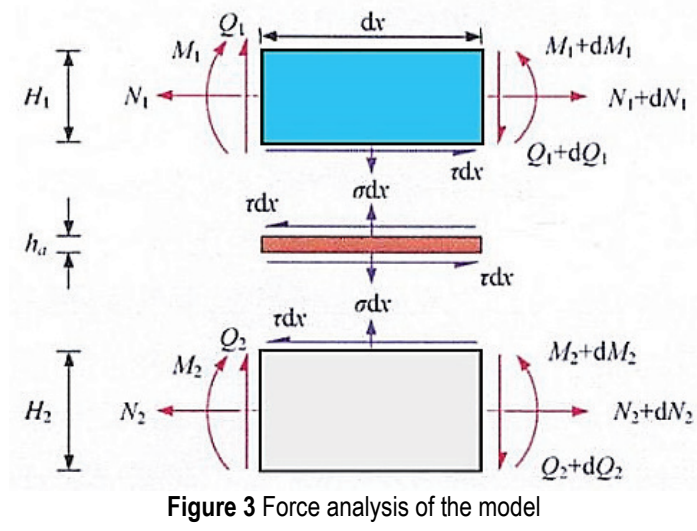

Where $A_{i}=A_{11}^{i}, C_{i}=k_{\mathrm{s}} A_{55}^{i}$ and $D_{i}=D_{11}^{i}$ are tensile, shear and bending stiffness of the bonded layer. For isotropic materials, they can be calculated by $A_{i}=E_{i}^{*} H_{i}$, $C_{i}=k_{\mathrm{s}} G_{i} H_{i} \quad$ and $\quad D_{i}=E_{i}^{*} H_{i}^{3} / 12, \quad$ in which $E_{i}^{*}=E_{i} /\left(1-v_{i}^{2}\right)$ and $G_{i}=E_{i} /\left[2\left(1+v_{i}\right)\right]$ is the effective elastic modulus and shear modulus of the bonded material under the assumption of plane strain, $k_{\mathrm{s}}$ represents Shear correction factor. The three internal forces of glass and adsorbent pad can be obtained by equilibrium equation respectively:

$$
\left\{\begin{array}{l}
\frac{\mathrm{d} N_{1}}{\mathrm{~d} x}+\tau=0, \frac{\mathrm{d} Q_{1}}{\mathrm{~d} x}+\sigma=0, \frac{\mathrm{d} M_{1}}{\mathrm{~d} x}+h_{1} \tau-Q_{1}=0 \\
\frac{\mathrm{d} N_{2}}{\mathrm{~d} x}-\tau=0, \frac{\mathrm{d} Q_{2}}{\mathrm{~d} x}-\sigma=0, \frac{\mathrm{d} M_{2}}{\mathrm{~d} x}+h_{2} \tau-Q_{2}=0
\end{array}\right.
$$

We define the corresponding flexibility parameter as $a_{i}=1 / A_{i}, \quad c_{i}=1 / C_{i}$ and $d_{i}=1 / D_{i}$. The relationship between displacement of glass and adsorbent pad and bond stress can be established as follows:

$$
\left\{\begin{array}{l}
\frac{\mathrm{d}^{2} u_{1}}{\mathrm{~d} x^{2}}=-a_{1} \tau, \frac{\mathrm{d} \varphi_{1}}{\mathrm{~d} x}+\frac{\mathrm{d}^{2} w_{1}}{\mathrm{~d} x^{2}}=-c_{1} \sigma, \frac{\mathrm{d}^{3} \varphi_{1}}{\mathrm{~d} x^{3}}=-d_{1} \sigma-d_{1} h_{1} \frac{\mathrm{d} \tau}{\mathrm{d} x} \\
\frac{\mathrm{d}^{2} u_{2}}{\mathrm{~d} x^{2}}=-a_{2} \tau, \frac{\mathrm{d} \varphi_{2}}{\mathrm{~d} x}+\frac{\mathrm{d}^{2} w_{2}}{\mathrm{~d} x^{2}}=-c_{2} \sigma, \frac{\mathrm{d}^{3} \varphi_{2}}{\mathrm{~d} x^{3}}=-d_{2} \sigma-d_{2} h_{2} \frac{\mathrm{d} \tau}{\mathrm{d} x}
\end{array}\right.
$$

\section{SOLUTION OF DIFFERENTIAL EQUATIONS 3.1 Differential Equation}

Both Eqs. (4) and (7) reflect the relationship between displacement of glass and adsorbent pad and bond stress. By combining the two formulas, the differential equation containing only one variable of stress is obtained:

$$
\left\{\begin{array}{l}
\frac{\mathrm{d}^{4} \sigma}{\mathrm{d} x^{4}}-k_{\sigma}\left(c_{1}+c_{2}\right) \frac{\mathrm{d}^{2} \sigma}{\mathrm{d} x^{2}}+k_{\sigma}\left(d_{1}+d_{2}\right) \sigma+k_{\sigma}\left(d_{1} h_{1}-d_{2} h_{2}\right) \frac{\mathrm{d} \tau}{\mathrm{d} x}=0 \\
\frac{\mathrm{d}^{3} \sigma}{\mathrm{d} x^{3}}-k_{\tau}\left(a_{2}+a_{1}+d_{2} h_{2}^{2}-d_{1} h_{1}^{2}\right) \frac{\mathrm{d} \tau}{\mathrm{d} x}-k_{\tau}\left(d_{1} h_{1}-d_{2} h_{2}\right) \sigma=0
\end{array}\right.
$$

\subsection{Solution of Bond Stress}

According to the first equation in Eq. (8), the first order differential of shear stress $\mathrm{d} \tau / \mathrm{d} x$ can be expressed as the expression of desorption stress. Then, the second equation can be substituted. After simplification, the sixth order homogeneous linear differential equation of desorption stress can be derived:

$\frac{\mathrm{d}^{6} \sigma}{\mathrm{d} x^{6}}+\eta_{1} \frac{\mathrm{d}^{4} \sigma}{\mathrm{d} x^{4}}+\eta_{2} \frac{\mathrm{d}^{2} \sigma}{\mathrm{d} x^{2}}+\eta_{3} \sigma=0$

in which:

$\left\{\begin{array}{l}\eta_{1}=-k_{\tau}\left(a_{1}+a_{2}+d_{1} h_{1}^{2}+d_{2} h_{2}^{2}\right)-k_{\sigma}\left(c_{1}+c_{2}\right) \\ \eta_{2}=k_{\tau} k_{\sigma}\left(a_{1}+a_{2}+d_{1} h_{1}^{2}+d_{2} h_{2}^{2}\right)\left(c_{1}+c_{2}\right)+k_{\sigma}\left(d_{1}+d_{2}\right)(10) \\ \eta_{3}=-k_{\tau} k_{\sigma}\left[\left(a_{1}+a_{2}\right)\left(d_{1}+d_{2}\right)+d_{1} d_{2}\left(h_{1}+h_{2}\right)^{2}\right]\end{array}\right.$

It can be seen that the characteristic equation of Eq. (9) is $\lambda^{6}+\eta_{1} \lambda^{4}+\eta_{2} \lambda^{2}+\eta_{3}=0$. If $\Lambda=\lambda^{2}$, the attributes of its solutions depend on $\Delta=q^{2} / 4+p^{3} / 27$, in which $p=\eta_{2}+\eta_{1}^{2} / 3$ and $q=2 \eta_{1}^{3} / 27-\eta_{1} \eta_{2} / 3+\eta_{3}$. It is assumed that the general form of the solution of desorption 
and shear stress is:

$$
\left\{\begin{array}{l}
\sigma(x)=\sum_{n=1}^{6} F_{n} \exp \left(\lambda_{n} x\right) \\
\tau(x)=-\frac{1}{d_{1} h_{1}-d_{2} h_{2}}\left\{\sum_{n=1}^{6} F_{n}\left[\frac{\lambda_{n}^{3}}{k_{\sigma}}-\left(c_{1}+c_{2}\right) \lambda_{n}+\frac{d_{1}+d_{2}}{\lambda_{n}}\right] \exp \left(\lambda_{n} x\right)+F_{7}\right\}
\end{array}\right.
$$

In the formula above, $F_{1}, F_{2}, \ldots, F_{7}$ are integral constants. It is necessary to calculate the boundary conditions by the internal force and displacement formula of the lower section.

\subsection{Internal Force and Displacement}

In the peeling process, there is a first order differential relationship between the internal force and the adsorptive force of the glass substrate, so the internal force and the expression of the bonded layer can be easily deduced by integral calculation of (11). By substituting (11) for the first set of equations in formula (6), and integrating variable $x$, the axial force can be obtained as follows:

$$
\left\{\begin{array}{l}
N_{1}(x)=\frac{\left\{\sum_{n=1}^{6} F_{n}\left[\frac{\lambda_{n}^{2}}{k_{\sigma}}-\left(c_{1}+c_{2}\right)+\frac{d_{1}+d_{2}}{\lambda_{n}^{2}}\right] \exp \left(\lambda_{n} x\right)+F_{7} x+F_{8}\right\}}{d_{1} h_{1}-d_{2} h_{2}} \\
N_{2}(x)=\frac{\left\{\sum_{n=1}^{6} F_{n}\left[\frac{\lambda_{n}^{2}}{k_{\sigma}}-\left(c_{1}+c_{2}\right)+\frac{d_{1}+d_{2}}{\lambda_{n}^{2}}\right] \exp \left(\lambda_{n} x\right)+F_{7} x+F_{11}\right\}}{d_{1} h_{1}-d_{2} h_{2}}
\end{array}\right.
$$

Similarly, there are transverse shear forces:

$$
\left\{\begin{array}{l}
Q_{1}(x)=-\left[\sum_{n=1}^{6} F_{n} \frac{1}{\lambda_{n}} \exp \left(\lambda_{n} x\right)+F_{9}\right] \\
Q_{2}(x)=\sum_{n=1}^{6} F_{n} \frac{1}{\lambda_{n}} \exp \left(\lambda_{n} x\right)+F_{12}
\end{array}\right.
$$

Finally, the expressions of stress (11) and shear force (13) are substituted into the third set of equations in formula (6) at the same time, and the bending moment can be calculated by an integral operation of variable $x$ :

$$
\begin{aligned}
& M_{1}(x)=\frac{h_{1}}{d_{1} h_{1}-d_{2} h_{2}}\left\{\begin{array}{l}
\sum_{n=1}^{6} F_{n}\left[\frac{\lambda_{n}{ }^{2}}{k_{\sigma}}-\left(c_{1}+c_{2}\right)+\frac{d_{2}\left(h_{1}+h_{2}\right)}{h_{1} \lambda_{n}{ }^{2}}\right] \exp \left(\lambda_{n} x\right)+ \\
+\left(F_{7}-\frac{d_{1} h_{1}-d_{2} h_{2}}{h_{1}} F_{9}\right) x+F_{10}
\end{array}\right\} \\
& M_{2}(x)=\frac{h_{2}}{d_{1} h_{1}-d_{2} h_{2}}\left\{\begin{array}{l}
\sum_{n=1}^{6} F_{n}\left[\frac{\lambda_{n}{ }^{2}}{k_{\sigma}}-\left(c_{1}+c_{2}\right)+\frac{d_{1}\left(h_{1}+h_{2}\right)}{h_{2} \lambda_{n}^{2}}\right] \exp \left(\lambda_{n} x\right)+ \\
+\left(F_{7}+\frac{d_{1} h_{1}-d_{2} h_{2}}{h_{2}} F_{12}\right) x+F_{13}
\end{array}\right\}
\end{aligned}
$$

Similarly, the axial and transverse displacement fields and corresponding rotation angles can be obtained. Through the above analysis and deduction, the mathematical expressions of all the basic parameters describing the bonding structure have been obtained, including the desorption stress $\sigma$, shear stress $\tau$, the internal forces $N_{1}, Q_{1}, M_{1}$ and displacement fields $u_{1}, \varphi_{1}$ and $w_{1}$ of the glass layer, and the internal forces $N_{2}, Q_{2}, M_{2}$ and displacement fields $u_{2}, \varphi_{2}$ and $w_{2}$ of the adsorption pad. The mechanical model of the mixed boundary of internal force and displacement field of bonded structure provided in this section is universal and has the potential to be applied in other scenarios to predict the internal stress of bonded structure.

\subsection{Integral Constant}

As mentioned above, 19 integral constants need to be determined by boundary conditions. Conventionally, each end surface of the upper and lower layers is determined by three boundary conditions (including force or displacement conditions), and a total of 12 boundary conditions are obtained for four end surfaces. This is obviously inconsistent with the total number of unknown integral constants. In fact, these integral constants are not independent of each other. Their internal relations can be derived by introducing the analytical expressions of bond stress and displacement into the formula (4). Since the left and right sides of the equal sign must be equal, the following internal relations of integral constants can be derived:

$$
\left\{\begin{array}{l}
F_{7}-d_{1} F_{9}-d_{2} F_{12}=0 \\
\left(a_{2}+a_{1}+d_{2} h_{2}^{2}+d_{1} h_{1}^{2}\right) F_{7}+\left(d_{1} h_{1}-d_{2} h_{2}\right)\left(d_{2} h_{2} F_{12}-d_{1} h_{1} F_{9}\right)=0 \\
a_{1} F_{8}+a_{2} F_{11}+d_{1} h_{1}^{2} F_{10}+d_{2} h_{2}^{2} F_{13}=0 \\
\left(d_{1} h_{1}-d_{2} h_{2}\right)\left(c_{1} F_{9}+c_{2} F_{12}\right)+d_{1} h_{1} F_{15}-d_{2} h_{2} F_{18}=0 \\
d_{1} h_{1} F_{10}-d_{2} h_{2} F_{13}=0 \\
a_{1} F_{14}+d_{1} h_{1}^{2} F_{15}+a_{2} F_{17}+d_{2} h_{2}^{2} F_{18}-F_{7} / k_{\tau}=0 \\
F_{16}-F_{19}=0
\end{array}\right.
$$

\begin{tabular}{|c|c|c|c|}
\hline Parameters & & Glass Substrate & Adsorption pad \\
\hline Material & & $\mathrm{Si}$ & Polvurethane \\
\hline \multirow{4}{*}{$\begin{array}{l}\text { Mechanical } \\
\text { properties }\end{array}$} & $\begin{array}{l}\text { Modulus of } \\
\text { elasticity }\end{array}$ & $7.09 \mathrm{E} 4$ & $\begin{array}{c}E_{x}=E_{z}=320 \\
E_{y}=30\end{array}$ \\
\hline & Poisson ratio & 0.23 & 0.4 \\
\hline & $\begin{array}{l}\text { Critical fracture } \\
\text { strength value } \\
(\mathrm{MPa})\end{array}$ & $157.2-72.2$ & - \\
\hline & $\begin{array}{l}\text { Critical bond } \\
\text { fracture energy } \\
(\mathrm{N} / \mathrm{mm})\end{array}$ & 0.08 & - \\
\hline \multirow{2}{*}{$\begin{array}{l}\text { Geometric } \\
\text { dimensions }\end{array}$} & Thickness (mm) & $0.2-1.0$ & 1.0 \\
\hline & Length $(\mathrm{mm})$ & $250-2200$ & 3000 \\
\hline \multirow{2}{*}{$\begin{array}{l}\text { Boundary } \\
\text { load } \\
\text { conditions }\end{array}$} & $\begin{array}{c}\text { Displacement } \\
\text { boundary }\end{array}$ & Free end & $\begin{array}{c}\text { Lower Surface } \\
\text { Fixation }\end{array}$ \\
\hline & loading method & $\begin{array}{c}\text { Left } \\
\text { concentrated } \\
\text { load } F=2 \mathrm{~N}\end{array}$ & - \\
\hline
\end{tabular}

Formula (2)-(15) shows that there are seven internal relations among the integral constants. With 12 independent boundary conditions under actual external loads, 19 linear equations about the integral constants can be obtained. Note that in practice, it is not necessary to solve all the integral constants, because some of them may not be solved due to the rigid body displacement of the whole or part of the structure in some cases. In fact, we only need to pay attention to the first seven integral constants contained in the bond stress formula.

Table 1 Geometric dimensions, material properties, loads and boundary conditions of the objects involved in the composite structure of glass substrates 


\section{EXPERIMENT}

\subsection{Model Parameters}

The geometric dimensions, material properties, loads and boundary conditions of all the objects involved in the computational model and experiment are shown in Tab. 1.

\subsection{Experiment Setup}

In order to verify the mechanical model proposed in this paper, the desorption process of glass substrates was experimented. The schematic diagram of the desorption process is shown in Fig. 4.

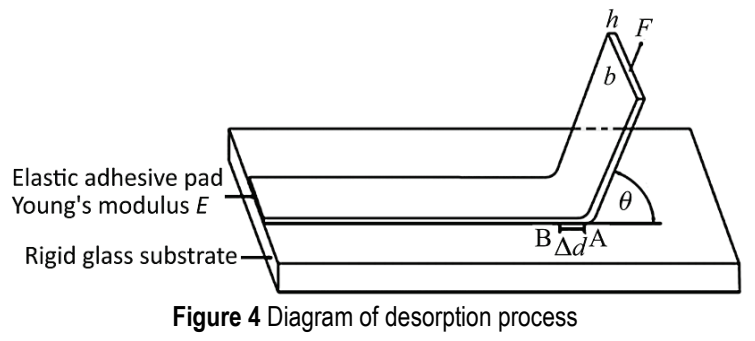

The desorption experiment was carried out on the experimental platform of NACHI six-axis manipulator with WACOH six-axis force sensor at the end as shown in Fig. 5. The maximum load of the manipulator is $4 \mathrm{kG}$ and the maximum working radius (arm length) is $541 \mathrm{~mm}$. The peeling angle can be adjusted from 0-180 and the peeling displacement can be set in the range of $0 \mathrm{~mm}$ to $200 \mathrm{~mm}$. The displacement accuracy of the manipulator is $0.01 \mathrm{~mm}$. In addition, desorption force $F$ is acquired by WEF-6A2004-RC24 WACOH six-axis force sensor in real time, and its accuracy can reach $0.01 \mathrm{~N}$. The desorption test refers to the Chinese standard GBT-7122-1996 high strength measurement floating roller method. The glass substrate is fixed on the table by glue. During the desorption experiment, the tensile strain of the specimens is kept within $10 \%$, which ensures that the linear elasticity hypothesis is valid. The elastic modulus $E$ of the specimens is $320 \mathrm{MPa}$, and the thickness and width of the specimens are $1.0 \mathrm{~mm}$ and $25 \mathrm{~mm}$ respectively.

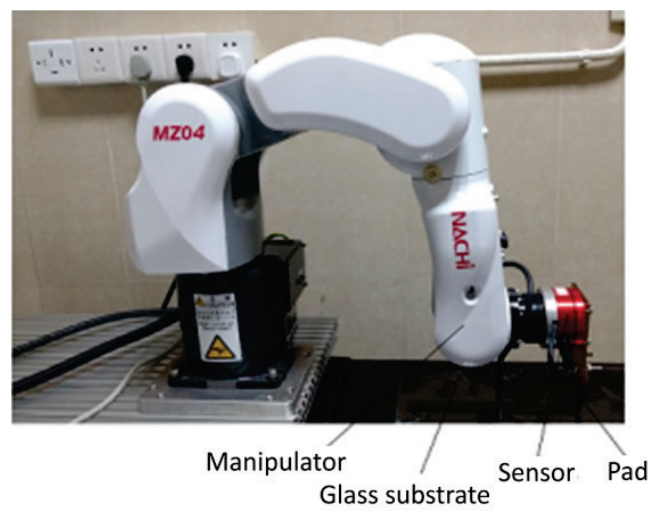

Figure 5 Desorption experiment platform

In order to keep the force angle unchanged in the experiment, it is necessary to understand the relationship between horizontal and vertical velocity. Fig. 6 shows the relationship between the horizontal and vertical velocity of the end effector of the manipulator when the desorption angles are acute and obtuse respectively.
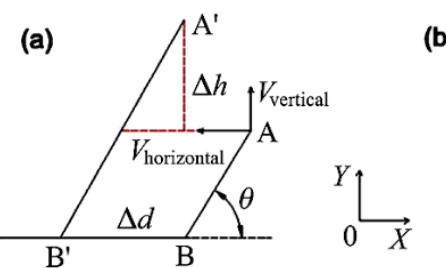

(b)

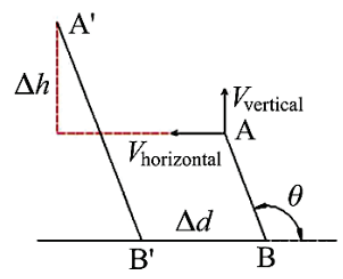

Figure 6 The relationship between the horizontal velocity $V_{\text {horizontal }}$ of the end effector and the vertical velocity $V_{\text {vertical }}$ at different peeling angles;

(a) $\theta$ is acute; (b) $\theta$ is obtuse

When $\theta$ is an acute angle, the crack extends from point $\mathrm{B}$ to point $\mathrm{B}^{\prime}$. In order to keep the desorption angle unchanged, the end effector must always keep along the $\overrightarrow{A A}$ ' direction. The horizontal displacement is $\Delta x=\Delta d-\Delta d \cos \theta=\Delta d(1-\cos \theta)$, the displacement in the vertical direction is $\Delta y=\Delta h=\Delta d \sin \theta$, the ratio of velocity in two directions equals the ratio of displacement:

$$
\frac{V_{\text {horizontal }}}{V_{\text {vertical }}}=\frac{\Delta d[1-\cos (\theta)]}{\Delta d \cdot \sin (\theta)}=\frac{1-\cos (\theta)}{\sin (\theta)}
$$

Similarly, when the angle $\theta$ is obtuse, the horizontal displacement is $\Delta x=\Delta d+\Delta d \cos \theta=\Delta d(1+\cos \theta)$, the displacement in the vertical direction is $\Delta y=\Delta h=\Delta d \sin \theta$, the ratio of velocity in two directions equals the ratio of displacement:

$\frac{V_{\text {horizontal }}}{V_{\text {vertical }}}=\frac{\Delta d[1+\cos (\theta)]}{\Delta d \cdot \sin (\theta)]}=\frac{1+\cos (\theta)}{\sin (\theta)}$

Whether the desorption angle is acute or obtuse, it can be found that the ratio of desorption velocity $V_{\text {desorption }}$ to vertical velocity is constant:

$$
\frac{V_{\text {desorption }}}{V_{\text {vertical }}}=\frac{\Delta d}{\Delta d \cdot \sin (\theta)}=\frac{1}{\sin (\theta)}
$$

In the design of desorption test, the horizontal and vertical velocity of the end effector of the manipulator can be calculated only by determining the desorption angle theta and the desorption speed $V_{\text {desorption }}$.

\subsection{Result and Analysis}

Fig. 7 shows the critical load $F_{\text {critical }}$ for desorption of glass substrates (thicknesses range from $0.2 \mathrm{~mm}$ to $1.0 \mathrm{~mm}$ ) from adsorption pad under mechanical analysis and experimental tests respectively. It can be seen from Fig. 7 that the analytical solution of the mechanical model presented in this paper is in good agreement with the experimental results. As the thickness of glass substrates increases, the critical load $F_{\text {critical }}$ decreases, which is consistent with that in reference [7]. This is because the overall stiffness of the system increases with the thickness of glass substrates, resulting in the decrease of bending deformation at the point to be desorbed, the decrease of stress intensity factor at the interface crack tip, and the decrease of critical load $F_{\text {critical }}$ from the relationship between energy release rate and stress intensity factor. 


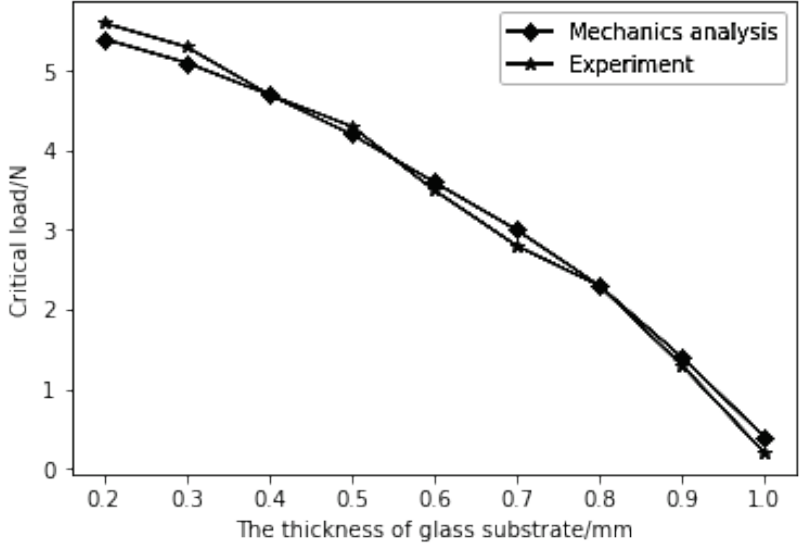

Figure7 Comparison of Desorption Critical Load between Mechanical Analysis and Experimental

\section{CONCLUSION}

The bonding mechanics model of glass substratesadsorbent pads proposed in this paper can effectively characterize the adsorbing effect of adsorbent pads on glass substrates, and can provide theoretical support for automatic non-destructive peeling of glass substrates. The difference between calculated and experimental desorption critical load is less than $7 \%$.

Although the mechanical model proposed in this paper is only applicable to the moment of desorption, which is analyzed as a steady state without considering the force on the interface between glass substrate and adsorbent pad after desorption, desorption is the core of non-destructive peeling of glass substrate, so the mechanical model can meet the needs of Engineering applications. The analytical method of mechanical model presented in this paper has certain universality. When the object is multi-layer bonded structure, whether under pure load or displacement boundary conditions, or under mixed force and position conditions, the model has general analytical solutions. The results can be used to predict the strength or analyze the bond fracture of multi-layer structure conveniently. In addition, the mechanical model can also be used to analyze the vacuum adsorption effect caused by material surface effect.

\section{Acknowledgments}

The work was supported by Industrial Commissioner Program of Changsha Science and Technology Bureau of Hunan Province (CSKJ2017-42) and the Fundamental Research Funds for the Central Universities of Central South University (2019zzts252).

\section{REFERENCES}

[1] Autumn, K., Liang, Y. A., Hsieh, S. T., Zesch, W., Chan, W. P., Kenny, T. W., Fearing, R., \& Full, R. J. (2000). Adhesive force of a single gecko foot-hair. Nature 2000, 405(6787), 681-685. https://doi.org/10.1038/35015073

[2] Geim, A. K., Dubonos, S. V., Grigorieva, I. V., et al. (2003). Microfabricated adhesive mimicking gecko foot-hair. Nature Materials, 2(7), 461-463. https://doi.org/10.1038/nmat917

[3] Maugis, D. \& Barquins, M. (1980). Fracture Mechanics and Adherence of Viscoelastic Solids. Journal of Physics D-
Applied Physics, 11(14), 1989. https://doi.org/10.1088/0022-3727/11/14/011

[4] Huguet, A. S. \& Barthel, E. (2000). Surface Forces and the Adhesive Contact of Axisymmetric Elastic Bodies. Journal of Adhesion, 74(1-4), 143-175. https://doi.org/10.1080/00218460008034528

[5] Sokolov, I., Ong, Q. K., Shodiev, H., et al. (2006). AFM study of forces between silica, silicon nitride and polyurethane pads. J Colloid Interface Sci, 300(2), 475-481. https://doi.org/10.1016/j.jcis.2006.04.023

[6] Goland, M. \& Reissner, E. (1944). The Stresses in Cemented Joints. De Velopments in Adhesives, 66.

[7] Bigwood, D. A. \& Crocombe, A. D. (1989). Elastic analysis and engineering design formulae for bonded joints. International Journal of Adhesion \& Adhesives, 9(4). 229242. https://doi.org/10.1016/0143-7496(89)90066-3

[8] Wang, J. \& Zeng, S. (2008). Thermal stresses analysis in adhesive/solder bonded biomaterial assemblies. Journal of Applied Physics, 104(11), 113508--8. https://doi.org/10.1063/1.3021357

[9] Yousefsani, S. A. \& Tahani, M. (2013). Analytical solutions for adhesively bonded composite single-lap joints under mechanical loadings using full layerwise theory. International Journal of Adhesion and Adhesives, 43(0), 3241. https://doi.org/10.1016/j.ijadhadh.2013.01.012

\section{Contact information}

\section{Liwei HOU}

Corresponding author

College of Mechanical and Electrical,

Central South University, Hunan 410083, China

Email: excharm@163.com

\section{Hengsheng WANG}

College of Mechanical and Electrical,

Central South University, Hunan 410083, China

State Key Laboratory for High Performance Complex Manufacturing,

Central South University, Hunan 410083, China

\section{Runhua LIU}

College of Mechanical and Electrical,

Central South University, Hunan 410083, China 